\title{
THE ASYMPTOTIC BEHAVIOR OF INTEGRABLE FUNCTIONS
}

\author{
CONSTANTIN P. NICULESCU AND FLORIN POPOVICI
}

\begin{abstract}
Given a density $d$ defined on the Borel subsets of $[0, \infty)$, the limit in density of a function $f:[0, \infty) \rightarrow \mathbb{R}$ is zero (abbreviated, $(d)$ $\left.\lim _{x \rightarrow \infty} f(x)=0\right)$ if there exists a set $S$ of zero density such that $f(x) \rightarrow 0$ as $x$ runs to $\infty$ outside $S$. It is proved that the behavior at infinity of every Lebesgue integrable function $f \in L^{1}(0, \infty)$ satisfies the relations $\left(d^{(n)}\right)$ $\lim _{x \rightarrow \infty}\left(\prod_{k=0}^{n} \ln ^{(k)} x\right) f(x)=0$, where $\left(d^{(n)}\right)_{n}$ is a scale of densities including the usual one, $d^{(0)}(A)=\lim _{r \rightarrow \infty} \frac{m(A \cap[0, r))}{r}$.
\end{abstract}

The analogy between convergent series and integrals over the positive semi-axis is an interesting topic from classical real analysis that flows continuously from the old days of mathematics to contemporary research. However, there is a fundamental property of convergent series in regard to which this analogy fails. Precisely, if $\sum a_{n}$ is a convergent series then $a_{n} \rightarrow 0$, but it is not always true, even when $f:[0, \infty) \rightarrow \mathbb{R}$ is positive, that if $\int_{0}^{\infty} f(x) d x$ is convergent then $f(x) \rightarrow 0$ as $x \rightarrow \infty$. An example is provided by the function

$$
f(x)= \begin{cases}1 & \text { for } x \in\left[n, n+1 / 2^{n}\right], n \in \mathbb{N} \\ 0 & \text { otherwise. }\end{cases}
$$

This example makes clear that in order to re-establish the analogy between series and integrals is necessary to consider a more general concept of limit at infinity that leaves off certain subsets of $\mathbb{R}_{+}$.

In order to put this in an abstract setting we will start with a family $\mathfrak{I}$ of measurable subsets of $\mathbb{R}_{+}$considered to be "small" or "negligible", that verifies the following four conditions:

$P I 1): \mathfrak{I}$ is closed under finite unions;

$P I 2): \mathfrak{I}$ is hereditary $(A \subset B \in \mathfrak{I}$ and $A$ measurable imply $A \in \mathfrak{I})$;

PI3): $\mathfrak{I}$ contains all bounded measurable subsets of $\mathbb{R}_{+}$;

$P I 4): \mathbb{R}_{+} \notin \mathfrak{I}$.

Such a family is called a proper ideal. We say that $\ell \in \mathbb{R}$ is the limit at infinity of a function $f:[a, \infty) \rightarrow \mathbb{R}$ modulo the proper ideal $\mathfrak{I}$, abbreviated,

$$
\ell=(\mathfrak{I})-\lim _{x \rightarrow \infty} f(x),
$$

if for every $\varepsilon>0$ there exists a subset $F_{\varepsilon} \in \mathfrak{I}$ outside which $|f(x)-\ell|<\varepsilon$.

Due to the property of heredity, the above concept of limit is equivalent to the fact that each of the sets $\{x:|f(x)-\ell| \geq \varepsilon\}$ belongs to I whenever $\varepsilon>0$.

Date: February 15, 2012.

2000 Mathematics Subject Classification. Primary 26A42; Secondary 37A45.

Key words and phrases. Lebesgue integral, density, convergence in density.

Published in Real Analysis Exchange, Vol. 38 (1), 2012/2013, 157-168. 
One can consider also the limit at infinity leaving a set $S$ in $\mathfrak{I}$,

$$
\ell=\lim _{\substack{x \rightarrow \infty \\ x \notin S}} f(x),
$$

with the meaning that $f(x) \rightarrow \ell$ as $x$ runs to $\infty$, outside $S$.

The usual limit at infinity corresponds to the case where $\mathfrak{I}$ is the proper ideal $\mathfrak{M}_{b}$ of all bounded measurable sets included in $\mathbb{R}_{+}$. In this case the two concepts of limit at infinity (1) and (2) are equivalent. In general only one implication works: the existence of limit (2) implies the limit (1).

Our problem mentioned above makes use of the ideal $\mathfrak{M}_{f}$, consisting of all measurable subsets of $\mathbb{R}_{+}$with finite measure.

The sum of a convergent series $\sum_{n \in \mathbb{N}} a_{n}$ is precisely the integral of the sequence of its terms with respect to the counting measure,

$$
c(A)=\text { number of elements in } A .
$$

Indeed,

$$
\sum_{n \in \mathbb{N}} a_{n}=\int_{\mathbb{N}} a_{n} d c(n) .
$$

The bounded subsets of $\mathbb{N}$ (endowed with the discrete metric) are the finite sets and thus they are the same with the subsets of $\mathbb{N}$ of finite measure. In the continuous case (that is, of $\mathbb{R}$ endowed with the Lebesgue measure $m$ ), there are sets of finite measure that are not bounded. It is precisely this fact that makes the difference between the behavior of series and integrals.

Theorem 1. If $f:[a, \infty) \rightarrow \mathbb{R}$ is a Lebesgue integrable function, then for every $\delta>0$ there exists a measurable subset $S$ of $[a, \infty)$ such that $m(S)<\delta$ and

$$
\lim _{\substack{x \rightarrow \infty \\ x \notin S}} f(x)=0 .
$$

Proof. Since $f$ is integrable, one can choose an increasing sequence $\left(a_{n}\right)_{n}$ of positive numbers such that

for every natural number $n$. Then the sets

$$
\int_{a_{n}}^{\infty}|f(x)| d x<\frac{1}{n^{3}}
$$

$$
S_{n}=\left\{x \in\left[a_{n}, a_{n+1}\right):|f(x)| \geq \frac{1}{n}\right\}
$$

are measurable and their union has finite measure because

$$
m\left(S_{n}\right)=n \int_{a_{n}}^{\infty} \frac{1}{n} \chi_{S_{n}}(x) d x \leq n \int_{a_{n}}^{\infty}|f(x)| d x \leq \frac{1}{n^{2}} .
$$

Therefore

$$
\lim _{x \rightarrow \infty} m(S \cap[x, \infty))=0,
$$

and thus by replacing $S$ by $S \cap\left[a_{N}, \infty\right)$ for $N$ large enough we may assume that $m(S)<\delta$.

Given $\varepsilon>0$, we denote by $n(\varepsilon)$ the smallest integer not less than $\max \{1 / \varepsilon, N\}$. Then for every $x$ in the set $\left[a_{n(\varepsilon)}, \infty\right) \backslash S$ we have

$$
|f(x)|<\frac{1}{n(\varepsilon)}<\varepsilon
$$

and the proof is done. 
A consequence of Theorem 1 is Barbălat's Lemma, an important tool for the analysis of the asymptotic behavior of nonlinear second order equations with forcing. See $[6]$.

Corollary 1. (Barbălat's Lemma [1]). If $f \in L^{1}([0, \infty)$ ) and $f$ is uniformly continuous, then $f(x) \rightarrow 0$ as $x \rightarrow \infty$.

Proof. Since $f$ is uniformly continuous, for $\varepsilon>0$ arbitrarily fixed there is $\delta(\varepsilon)>$ 0 such that

$$
x, y \in[0, \infty),|x-y|<\delta(\varepsilon) \Rightarrow|f(x)-f(y)|<\frac{\varepsilon}{2} .
$$

According to Theorem 1, there is a measurable subset $S$ with $m(S)<\delta(\varepsilon) / 2$ outside which $f(x) \rightarrow 0$ as $x \rightarrow \infty$. This gives us an $x_{\varepsilon}$ such that $|f(x)|<\varepsilon / 2$ for every $x \in\left[x_{\varepsilon}, \infty\right) \backslash S$.

As $S$ has finite measure, it admits a covering $\left(I_{n}\right)_{n}$ consisting of pairwise disjoint open intervals such that $\sum_{n} m\left(I_{n}\right)<\delta(\varepsilon)$.

Therefore, if $x \in\left[x_{\varepsilon}, \infty\right) \cap S$, then necessarily $x$ belongs to an interval $\left[x_{\varepsilon}+\right.$ $\left.k \delta(\varepsilon), x_{\varepsilon}+(k+1) \delta(\varepsilon)\right)$ for some $k \in \mathbb{N}$. Since the length of this interval is precisely $\delta(\varepsilon)$, it must contain elements $y \geq x_{\varepsilon}$ not in $S$. Then

$$
|f(x)| \leq|f(x)-f(y)|+|f(y)|<\frac{\varepsilon}{2}+\frac{\varepsilon}{2}=\varepsilon,
$$

and the proof is done.

It is worth to notice that while Barbălat's Lemma remains valid in the context of improper Riemann integrability, the conclusion of Theorem 1 fails such an extension. An example is provided by the zigzag function $f:[0, \infty) \rightarrow \mathbb{R}$ joining the points $(0,0),\left(\frac{1}{2}, 1\right),(1,0),\left(1+\frac{1}{4},-1\right),\left(1+\frac{1}{2}, 0\right)$, and so on. A series representation of this function can be obtained by considering the tent function of base $[a, b]$ :

$$
T_{[a, b]}(x)=\frac{2}{b-a} \min \{x-a, b-x\} \chi_{[a, b]}(x), \quad x \in \mathbb{R} .
$$

Indeed,

$$
f(x)=T_{[0,1]}(x)-T_{\left[1,1+\frac{1}{2}\right]}(x)+T_{\left[1,1+\frac{1}{2}\right]}(x)-T_{\left[1+\frac{1}{2}, 1+\frac{1}{2}+\frac{1}{3}\right]}(x)+\cdots .
$$

Theorem 1 is just the top of the iceberg. In our recent paper [7] we were able to deepen the similarity between series and integrals by considering weighted limits associated to a scale of proper ideals

$$
\mathfrak{I}^{(0)} \subset \mathfrak{I}^{(1)} \subset \mathfrak{I}^{(2)} \subset \cdots,
$$

each of them consisting of the sets where a certain density vanishes. The densities are aimed to measure how thin are the various Borel subsets of $\mathbb{R}_{+}$. The concept of set of zero density was first considered by B. O. Koopman and J. von Neumann [4] in a famous paper dedicated to weakly mixing transformations.

The purpose of the present note is to improve the main result in [7] and to offer a much simpler argument.

In order to smooth our presentation we will adopt the convention used in dynamical system theory for the iterates of a function $f=f(x)$ :

$$
f^{(0)}(x)=x \text { and } f^{(n)}(x)=(\underbrace{f \circ f \circ \cdots \circ f}_{n \text { times }})(x) \text { for } n \geq 1 .
$$


The density of order 0 (the usual density) is defined by the formula

$$
\begin{aligned}
d^{(0)}(A) & =\lim _{r \rightarrow \infty} \frac{1}{r} \int_{A \cap[0, r)} d t \\
& =\lim _{r \rightarrow \infty} \frac{m(A \cap[0, r))}{r} .
\end{aligned}
$$

This corresponds to the limiting relative frequency in probability theory. The next density in our scale, the density of order 1, is nothing but the continuous analogue of harmonic density from number theory,

$$
d^{(1)}(A)=\lim _{x \rightarrow \infty} \frac{1}{\ln x} \int_{A \cap[1, x)} \frac{d t}{t} .
$$

See [3], p. 241. Sometimes $d^{(0)}$ is denoted as $d$, and $d^{(1)}$ as $d_{h}$. These two densities are the first terms of the following scale of densities,

$$
d^{(n)}(A)=\lim _{x \rightarrow \infty} \frac{1}{\ln ^{(n)} x} \int_{A \cap\left[\exp ^{(n-1)} 1, x\right)} d \alpha_{n}, \quad n \geq 0,
$$

where $\exp ^{(-1)} 1=0$ and

$$
d \alpha_{0}=d t \text { and } d \alpha_{n}=\frac{d t}{\prod_{k=0}^{n-1} \ln ^{(k)} t} \quad \text { for } n \geq 1
$$

Given a real-valued function $f$ defined on an interval $[a, \infty)$, its limit in density of order $n$ at infinity,

$$
\ell=\left(d^{(n)}\right)-\lim _{x \rightarrow \infty} f(x)
$$

is defined as the limit modulo the proper ideal of all sets of zero density of order $n$.

Lemma 1. $d^{(n)}(A)=0$ implies $d^{(n+1)}(A)=0$, and thus the existence of limit in density of order $n$ implies the existence of limit in density of order $n+1$.

Proof. We will consider here the case where $n \geq 1$. The case where $n=0$ can be treated similarly.

Let $\varepsilon>0$. Since $d^{(n)}(A)=0$ one can choose a number $s \geq \exp ^{(n-1)} 1$ such that

$$
\frac{1}{\ln ^{(n)} x} \int_{A \cap\left[\exp ^{(n-1)} 1, x\right)} \frac{d t}{\prod_{k=0}^{n-1} \ln ^{(k)} t}<\frac{\varepsilon}{3}
$$


for every $x \geq s$. Then for $x>\max \left\{s, \exp ^{(n)} 1, \exp ^{(n)}\left(\frac{3}{\varepsilon} \ln ^{(n+1)} s\right)\right\}$ we have

$$
\begin{gathered}
0 \leq \frac{1}{\ln ^{(n+1)} x} \int_{A \cap\left[\exp ^{(n)} 1, x\right)} \frac{d t}{\prod_{k=0}^{n} \ln ^{(k)} t} \\
=\frac{1}{\ln ^{(n+1)} x} \int_{A \cap\left[\exp ^{(n)} 1, s\right)} \frac{d t}{\prod_{k=0}^{n} \ln ^{(k)} t} \\
\quad+\frac{1}{\ln ^{(n+1)} x} \int_{A \cap[s, x)} \frac{d t}{\prod_{k=0}^{n} \ln ^{(k)} t} \\
\leq \frac{\ln ^{(n+1)} s}{\ln ^{(n+1)} x}+\frac{1}{\ln ^{(n+1)} x} \int_{s}^{x} \frac{1}{\ln ^{(n)} t} \frac{d}{d t}\left(\int_{s}^{t} \frac{\chi_{A \cap[s, x)}(t) d \tau}{\prod_{k=0}^{n-1} \ln ^{(k)} \tau}\right) d t \\
<\frac{\varepsilon}{3}+\frac{1}{\ln ^{(n+1)} x}\left(\frac{1}{\ln ^{(n)} x} \int_{s}^{x} \frac{\chi_{A \cap[s, x)}(t) d \tau}{\prod_{k=0}^{n-1} \ln ^{(k)} \tau}+\right. \\
\left.+\int_{s}^{x} \frac{1}{\left(\ln ^{(n)} t\right)^{2} \prod_{k=0}^{n-1} \ln ^{(k)} t}\left(\int_{s}^{t} \frac{\chi_{A \cap[s, x)}(t) d \tau}{\prod_{k=0}^{n-1} \ln ^{(k)} \tau}\right) d t\right) \\
<\frac{\varepsilon}{3}+\frac{\varepsilon}{3 \ln ^{(n+1)} x}+\frac{\varepsilon}{3 \ln ^{(n+1)} x} \int_{s}^{x} \frac{d t}{\prod_{k=0}^{n} \ln ^{(k)} t} \\
<\frac{2 \varepsilon}{3}+\frac{\varepsilon}{3 \ln ^{(n+1)} x}\left(\ln ^{(n+1)} x-\ln ^{(n+1)} s\right)<\varepsilon
\end{gathered}
$$

and the proof is done.

Another notable fact is the equivalence of limit in density of order $n$ with that of limit at infinity leaving a set of zero density of order $n$.

Theorem 2. For every measurable function $f:[a, \infty) \rightarrow \mathbb{R}$ the following two conditions are equivalent:

i) $\left(d^{(n)}\right)-\lim _{x \rightarrow \infty} f(x)=0$;

ii) there exists a subset $S \subset[a, \infty)$ of zero density of order $n$, such that

$$
\lim _{\substack{x \rightarrow \infty \\ x \notin S}} f(x)=0 .
$$

Proof. The implication $i i) \Rightarrow i$ ) is clear. We will detail the implication $i) \Rightarrow i i$ ) in the case where $n \geq 1$ (the argument when $n=0$ being similar).

According to our hypothesis each of the sets

$$
S_{\varepsilon}=\{x \in[a, \infty):|f(x)| \geq \varepsilon\}, \quad \varepsilon>0,
$$

has zero density of order $n$. Since $d^{(n)}\left(S_{1}\right)=0$, one can choose an element $x_{1} \in$ $[a, \infty) \cap\left[\exp ^{(n-1)} 1, \infty\right)$ such that

$$
\frac{1}{\ln ^{(n)} x} \int_{S_{1} \cap\left[\exp ^{(n-1)} 1, x\right)} d \alpha_{n}<1
$$

for every $x>x_{1}$. Since $d^{(n)}\left(S_{1 / 2}\right)=0$, one can choose an element $x_{2}>x_{1}$ such that

$$
\frac{1}{\ln ^{(n)} x} \int_{S_{1 / 2} \cap\left[\exp ^{(n-1)} 1, x\right)} d \alpha_{n}<\frac{1}{2}
$$


for every $x>x_{2}$. By induction one obtains a strictly increasing sequence $\left(x_{k}\right)_{k}$ such that $\lim _{k \rightarrow \infty} x_{k}=\infty$ and

$$
\frac{1}{\ln ^{(n)} x} \int_{S_{1 / k} \cap\left[\exp ^{(n-1)} 1, x\right)} d \alpha_{n}<\frac{1}{k}
$$

for all $x>x_{k}$. Consider the set

$$
S=\bigcup_{k=1}^{\infty}\left(S_{1 / k} \cap\left[x_{k}, x_{k+1}\right)\right) .
$$

We will show that $d^{(n)}(S)=0$ and $\left.\lim _{x \rightarrow \infty} f\right|_{[a, \infty) \backslash S}=0$.

In fact, for $\varepsilon>0$ arbitrarily fixed put $N=\lfloor 1 / \varepsilon\rfloor+1$. Then every $x \geq x_{N}$ lies in an interval $\left[x_{p}, x_{p+1}\right)$, whence

$$
\begin{aligned}
S \cap[a, x) & =\left(\bigcup_{k=1}^{\infty}\left(S_{1 / k} \cap\left[x_{k}, x_{k+1}\right)\right)\right) \cap[a, x) \\
& =\left(\bigcup_{k=1}^{p}\left(S_{1 / k} \cap\left[x_{k}, x_{k+1}\right)\right)\right) \cap[a, x) \\
& \subset S_{1 / p} \cap\left[x_{1}, x\right) \subset S_{1 / p} \cap[a, x) .
\end{aligned}
$$

Therefore for every $x \geq x_{N}$ we get

$$
\frac{1}{\ln ^{(n)} x} \int_{S \cap\left[\exp ^{(n-1)} 1, x\right)} d \alpha_{n}
$$

$$
\leq \frac{1}{\ln ^{(n)} x} \int_{S_{1 / p} \cap\left[\exp ^{(n-1)} 1, x\right)} d \alpha_{n}<\frac{1}{p} \leq \frac{1}{N}<\varepsilon,
$$

and thus $d^{(n)}(S)=0$.

Since every $x \in\left[x_{N}, \infty\right) \backslash S$ belongs to a set $\left[x_{k}, x_{k+1}\right) \backslash S_{1 / k}$ for some $k \geq N$, it follows that

$$
|f(x)|<\frac{1}{k} \leq \frac{1}{N}<\varepsilon
$$

and thus $\lim _{x \rightarrow \infty, x \notin S} f(x)=0$. The proof is done.

We state now the main result of our paper.

Theorem 3. If $f \in L^{1}(0, \infty)$, then

$$
\left(d^{(n)}\right)-\lim _{x \rightarrow \infty}\left(\prod_{k=0}^{n} \ln ^{(k)} x\right) f(x)=0
$$

for every $n \in \mathbb{N}$.

B. O. Koopman and J. von Neumann [4] have introduced the concept of convergence in density in connection with the convergence of certain weighted arithmetic means. More precisely, they proved that every locally integrable function $f:[0, \infty) \rightarrow \mathbb{R}$ that verifies the condition

$$
\lim _{x \rightarrow \infty} \frac{1}{x} \int_{0}^{x}|f(t)| d t=0
$$

verifies also the condition $\left(d^{(0)}\right)-\lim _{x \rightarrow \infty} f(x)=0$. This fact can be extended to densities of all orders. 
Lemma 2. Suppose that $f:[0, \infty) \rightarrow \mathbb{R}$ is a locally integrable function and $n$ is a positive integer. Then

$$
\lim _{x \rightarrow \infty} \frac{1}{\ln ^{(n)} x} \int_{\exp ^{(n-1)} 1}^{x}|f(t)| d \alpha_{n}=0 \text { implies }\left(d^{(n)}\right)-\lim _{x \rightarrow \infty} f(x)=0,
$$

and the converse holds if in addition $f$ is bounded.

Proof. The first assertion follows by reductio ad absurdum. Indeed, according to Theorem 2, if $\left(d^{(n)}\right)-\lim _{x \rightarrow \infty} f(x)=0$ fails, then for some $\varepsilon_{0}>0$ the set

$$
S_{\varepsilon_{0}}=\left\{x \in\left[\exp ^{(n-1)} 1, \infty\right):|f(x)| \geq \varepsilon_{0}\right\}
$$

does not have zero density of order $n$. Consequently there exist a positive number $C$ and an increasing sequence $\left(x_{k}\right)_{k}$ of elements of $\left(\exp ^{(n-1)} 1, \infty\right)$ such that $\lim _{k \rightarrow \infty} x_{k}=\infty$ and

for every $k$. Then

$$
\frac{1}{\ln ^{(n)} x_{k}} \int_{S_{\varepsilon_{0}} \cap\left[\exp ^{(n-1)} 1, x_{k}\right)} d \alpha_{n} \geq C
$$

$$
\begin{aligned}
\frac{1}{\ln ^{(n)} x_{k}} \int_{\exp ^{(n-1)} 1}^{x_{k}} \mid & |f(t)| d \alpha_{n} \\
& \geq \frac{1}{\ln ^{(n)} x_{k}} \int_{S_{\varepsilon_{0}} \cap\left[\exp ^{(n-1)} 1, x_{k}\right)}|f(t)| d \alpha_{n} \\
& \geq \frac{\varepsilon_{0}}{\ln ^{(n)} x_{k}} \int_{S_{\varepsilon_{0}} \cap\left[\exp ^{(n-1)} 1, x_{k}\right)} d \alpha_{n} \geq C \varepsilon_{0},
\end{aligned}
$$

which contradicts our hypothesis.

As concerns the second assertion, assume $\left(d^{(n)}\right)-\lim _{x \rightarrow \infty} f(x)=0$ and fix arbitrarily $\varepsilon>0$. Since the set $S_{\varepsilon}=\left\{x \in\left[\exp ^{(n-1)} 1, \infty\right):|f(x)| \geq \varepsilon / 2\right\}$ has zero density of order $n$, there must exist a number $x_{\varepsilon} \geq \exp ^{(n-1)} 1$ such that

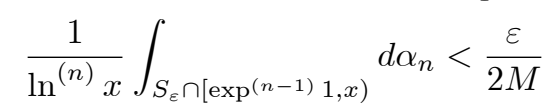

for every $x>x_{\varepsilon}$. Here $M=\sup _{x \geq 0}|f(x)|$. Then for $x>x_{\varepsilon}$ we have

$$
\begin{aligned}
0 \leq \frac{1}{\ln ^{(n)} x} \int_{\exp ^{(n-1)} 1}^{x} & |f(t)| d \alpha_{n} \\
\leq & \frac{1}{\ln ^{(n)} x} \int_{\left[\exp ^{(n-1)} 1, x\right) \backslash S_{\varepsilon}}|f(t)| d \alpha_{n} \\
& +\frac{1}{\ln ^{(n)} x} \int_{\left[\exp ^{(n-1)} 1, x\right) \cap S_{\varepsilon}}|f(t)| d \alpha_{n} \\
& <\frac{\varepsilon}{2}+M \cdot \frac{\varepsilon}{2 M}=\varepsilon
\end{aligned}
$$

and the proof is done.

Lemma 3. If $\omega:[a, \infty) \rightarrow \mathbb{R}$ is a nonincreasing, differentiable, and bounded function and $f:[a, \infty) \rightarrow \mathbb{R}$ is a function locally integrable with respect to $\omega d \alpha_{n}$, then

$$
\lim _{x \rightarrow \infty} \omega(x) \int_{a}^{x} f(t) d \alpha_{n}=0 .
$$


Proof. The nontrivial case is when $\omega(x)>0$ for every $x$. Given $\varepsilon>0$, one can choose $y>0$ such that

$$
\left|\int_{y}^{x} \omega(t) f(t) d \alpha_{n}\right|<\frac{\varepsilon}{3} \quad \text { for every } x>y .
$$

According to the formula of integration by parts for absolutely continuous functions,

$$
\begin{aligned}
& \omega(x) \int_{a}^{x} f(t) d \alpha_{n}=\omega(x) \int_{a}^{x} f(t) \frac{d t}{\prod_{k=0}^{n-1} \ln ^{(k)} t} \\
& =\omega(x)\left(\int_{a}^{y} f(t) \frac{d t}{\prod_{k=0}^{n-1} \ln ^{(k)} t}+\int_{y}^{x} \frac{1}{\omega(t)} \omega(t) f(t) \frac{d t}{\prod_{k=0}^{n-1} \ln ^{(k)} t}\right) \\
& =\omega(x)\left(\int_{a}^{y} f(t) \frac{d t}{\prod_{k=0}^{n-1} \ln ^{(k)} t}+\int_{y}^{x} \frac{1}{\omega(t)} \frac{d}{d t}\left(\int_{y}^{t} \omega(s) f(s) \frac{d s}{\prod_{k=0}^{n-1} \ln ^{(k)} s}\right) d t\right) \\
& =\omega(x)\left(\int_{a}^{y} f(t) \frac{d t}{\prod_{k=0}^{n-1} \ln ^{(k)} t}+\frac{1}{\omega(x)} \int_{y}^{x} \omega(s) f(s) \frac{d s}{\prod_{k=0}^{n-1} \ln ^{(k)} s}\right. \\
& \left.\quad-\int_{y}^{x} \frac{\omega^{\prime}(t)}{\omega^{2}(t)}\left(\int_{y}^{t} \omega(s) f(s) \frac{d s}{\prod_{k=0}^{n-1} \ln ^{(k)} s}\right) d t\right) .
\end{aligned}
$$

On the other hand, for $x>y$ sufficiently large we have

$$
\begin{aligned}
& \mid \omega(x) \int_{a}^{y} f(t) \frac{d t}{\prod_{k=0}^{n-1} \ln ^{(k)} t}+\int_{y}^{x} \omega(s) f(s) \frac{d s}{\prod_{k=0}^{n-1} \ln ^{(k)} s} \mid \\
& \leq \omega(x)\left|\int_{a}^{y} f(t) \frac{d t}{\prod_{k=0}^{n-1} \ln ^{(k)} t}\right|+\frac{\varepsilon}{3} \leq \frac{2 \varepsilon}{3},
\end{aligned}
$$

and

$$
\begin{gathered}
\left|\omega(x) \int_{y}^{x} \frac{\omega^{\prime}(t)}{\omega^{2}(t)}\left(\int_{y}^{t} \omega(s) f(s) \frac{d s}{\prod_{k=0}^{n-1} \ln ^{(k)} s}\right) d t\right| \\
\leq \omega(x) \int_{y}^{x}\left|\frac{\omega^{\prime}(t)}{\omega^{2}(t)}\left(\int_{y}^{t} \omega(s) f(s) \frac{d s}{\prod_{k=0}^{n-1} \ln ^{(k)} s}\right)\right| d t \\
\leq \frac{\varepsilon}{3} \omega(x) \int_{y}^{x} \frac{-\omega^{\prime}(t)}{\omega^{2}(t)} d t \\
\quad \leq \frac{\varepsilon}{3} \omega(x)\left(\frac{1}{\omega(y)}-\frac{1}{\omega(x)}\right) \leq \frac{\varepsilon}{3},
\end{gathered}
$$

whence $\left|\omega(x) \int_{a}^{x} f(t) d \alpha_{n}\right| \leq \varepsilon$ for $x$ sufficiently large.

We are now in a position to detail the proof of Theorem 3. The function $f$ being integrable on $[a, \infty)$, it follows that the product $\left(\prod_{k=0}^{n} \ln ^{(k)} x\right) f$ is locally integrable with respect to the measure $\omega(x) d \alpha_{n}$, where $\omega(x)=1 / \ln ^{(n)} x$. According to Lemma 3,

$$
\lim _{x \rightarrow \infty} \frac{1}{\ln ^{(n)} x} \int_{a}^{x}\left(\prod_{k=0}^{n} \ln ^{(k)} x\right) f(t) \frac{d t}{\prod_{k=0}^{n-1} \ln ^{(k)} t}=0
$$


so by Lemma 2 we conclude that $\left(d^{(n)}\right)-\lim _{x \rightarrow \infty}\left(\prod_{k=0}^{n} \ln ^{(k)} x\right) f(x)=0$.

A different approach of the behavior at infinity of an integrable function has been recently described by E. Lesigne [5].

It is worth to notice here the existence of a discrete companion of Theorem 3, working for positive series. The details are the same, with the difference that the role of Lebesgue measure over $[0, \infty)$ is taken by the counting measure over the nonnegative integers.

Acknowledgement. This work is supported by a grant of the Romanian National Authority for Scientific Research, CNCS - UEFISCDI, project number PN-II-IDPCE-2011-3-0257.

\section{REFERENCES}

[1] I. Barbălat, Systemes d'équations différentielle d'oscillations nonlinéaires, Rev. Roumaine Math. Pures Appl. 4 (1959) 267-270.

[2] H. Furstenberg, Recurrence in Ergodic Theory and Combinatorial Number Theory, Princeton University Press, Princeton, New Jersey, 1981.

[3] H. Halberstam and K.F. Roth, Sequences, 2nd Ed., Springer-Verlag, New York, 1983.

[4] B.O. Koopman and J. von Neumann, Dynamical systems of continuous spectra, Proc. Natl. Acad. Sci. U.S.A. 18 (1932) 255-263.

[5] E. Lesigne, On the Behavior at Infinity of an Integrable Function, The American Mathematical Monthly 117 (2010) 175-181.

[6] H. Logemann and E.P. Ryan, Asymptotic Behavior of Nonlinear Systems, The American Mathematical Monthly 111 (2004) 864-889.

[7] C.P. Niculescu and F. Popovici, A note on the behavior of integrable functions at infinity, J. Math. Anal. Appl. 381 (2011) 742-747.

[8] T. Salát and V. Toma, A classical Olivier's theorem and statistically convergence, Annales Math. Blaise Pascal 10 (2003) 305-313.

University of Craiova, Department of Mathematics, Craiova RO-200585, Romania

E-mail address: cniculescu47@yahoo.com

College Grigore Moisil, Braşov, Romania

E-mail address: popovici.florin@yahoo.com 